УДК 539.2

\title{
ВЗАИМОДЕЙСТВИЕ ЛОКАЛИЗОВАННЫХ СОСТОЯНИЙ ВБЛИЗИ ГРАНИЦЫ РАЗДЕЛА НЕЛИНЕЙНЫХ СРЕД
}

\author{
(C) 2017 С. Е. Савотченко \\ Белгородский государственный технологический университет имени В. Г. Шухова, ул. Костюкова, 46, \\ 308012 Белгород, Россия \\ e-mail: savotchenkose@mail.ru
}

Поступила в редакцию: 24.01.2017 г.

\begin{abstract}
Аннотация. В работе рассмотрены связанные состояния, локализованные вблизи границы раздела нелинейных сред и свободно распространяющиеся вдоль нее. Задача сводится к решению нелинейного уравнения Шредингера с граничными условиями особого вида. Установлено, что возможно существование нелинейных локализованных состояний, несимметричных относительно границы раздела сред. Структура и форма таких состояний определяется знаком ангармонизма взаимодействия в среде и интенсивностью взаимодействия возбуждений и другими характеристиками среды. Получены соотношения, определяющие значения энергии локализованной вдоль границы раздела сред волне при фиксированном направлении ее волнового вектора.
\end{abstract}

Ключевые слова: нелинейное уравнение Шредингера, плоский дефект, солитон, локализованные состояния, нелинейные волны.

Большую роль в квантовой электронике играют закономерности локализации различных возбуждений вблизи границ раздела нелинейных сред. Например, особенности локализацияиэлектромагнитных волн вблизи границ раздела нелинейных сред с использованием теории солитонов были описаны в $[1,2]$, где показано, что возможно существование нелинейных локализованных волн с несимметричным профилем, отличающихся от свободно распространяющихся солитонов и называемых нелинейными поверхностными волнами.

Часто возникает необходимость разработки моделей, позволяющих аналитически описывать на качественном уровне эффекты локализации возбуждений, обусловленных характером взаимодействия их с дефектами, причем как в линейном приближении, учитывающим только гармоническое взаимодействие атомов среды, так в нелинейных средах, где учитываются ангармонизмы межатомных связей. Обычно для теоретического описания таких явлений привлекались модели, в которых дефект описывался короткодействующим потенциалом [3, 4].

Нелинейное уравнение Шредингера (НУШ) широко применяется для построения математических моделей сред с дефектами $[5,6]$. Как было показа- но в [7], НУШ может описывать состояния полей различной физической природы: упругого, электрического и магнитного. Поэтому для описания новых эффектов, связанных с локализацией возбуждений различной физической природы вблизи дефектов, имеет смысл далее рассматривать математическую модель, использующую НУШ, которому подчиняется функция $\psi$, выступающая в роли огибающей комплексного поля компонент вектора намагниченности в легкоосном ферромагнетике, либо комплексной амплитуды упругого поля смещения сдвиговой волны в кубическом кристалле с плоским дефектом, либо комплексной функции из амплитуд компонент электрического поля в оптической нелинейной среде [8]. Тогда параметры в уравнении будут иметь соответствующий физических смысл в рамках одной из трех указанных моделей.

Будем считать, что частица имеет сложный линейный закон дисперсии (зависимость энергии $E$ от волнового вектора $\left.\mathbf{k}=\left(k_{x}, k_{y}, k_{z}\right)\right)$, с несколькими ветвями, отличающимися параметрами. Ограничимся рассмотрением взаимодействия двух соседних зон спектра. Такое взаимодействие возможно за счет наличия дефекта, в качестве которого будем рассматривать границу раздела сред, отличающихся параметрами ангармонизма межатомного взаи- 


\section{C. Е. САВОТЧЕНКО}

модействия. Данная граница раздела, как плоский дефект, будет создавать возмущение характеристик среды, которое сосредоточено на расстояниях, существенно меньших ширины локализации распространяющихся волн.

Пусть граница раздела сред расположена в плоскости $x O y$, перпендикулярно оси $O z$. Будем считать, что ангармонизм среды отличается по разные стороны от границы раздела, а также ветви $j=1,2$ возбуждений характеризуются различной по абсолютной величине нелинейностью:

$$
\gamma_{j}(z)=\left\{\begin{array}{c}
\gamma_{j}^{(-)}, z<0 \\
\gamma_{j}^{(+)}, z>0
\end{array}\right.
$$

В линейной среде без дефекта частица имеет закон дисперсии с двумя ветвями, вблизи дна зон которых они описываются квадратичными формами:

$$
E=\frac{k_{x}^{2}+k_{y}^{2}+k_{z}^{2}}{2 m_{1}}, E=\Omega+\frac{k_{y}^{2}+k_{z}^{2}}{2 m_{2}}+\frac{\left(k_{x}-k_{0}\right)^{2}}{2 m_{3}},
$$

$m_{j}(j=1,2,3), k_{0}, \Omega$ - параметры, определяющие форму ветвей закона дисперсии вблизи дна двух соседних зон (принято $\hbar=1)$.

Волновые функции стационарных состояний $\psi_{j}(x, \underline{y}, z), j=1,2$, отвечающие двум рассматриваемым ветвям закона дисперсии, в среде без дефекта описывают независимые состояния, представляющие собой свободно распространяющиеся солитоны. При наличии границы раздела сред в качестве модели для описания особенностей взаимодействия предлагается использовать трехмерные нелинейные уравнения Шредингера, которым подчиняются данные волновые функции:

$$
\begin{gathered}
\frac{1}{2 m_{1}} \Delta \psi_{1}+E \psi_{1}+\gamma_{1}(z)\left|\psi_{1}\right|^{2} \psi_{1}=U_{1}, \\
\frac{1}{2 m_{2}}\left(\frac{\partial^{2} \psi_{2}}{\partial x^{2}}+\frac{\partial^{2} \psi_{2}}{\partial y^{2}}\right)+\frac{1}{2 m_{3}}\left(\frac{\partial}{\partial z}-i k_{0}\right)^{2} \psi_{2}+ \\
+(E-\Omega) \psi_{2}+\gamma_{2}(z)\left|\psi_{2}\right|^{2} \psi_{2}=U_{2},
\end{gathered}
$$

где $\Delta=\frac{\partial^{2}}{\partial x^{2}}+\frac{\partial^{2}}{\partial y^{2}}+\frac{\partial^{2}}{\partial z^{2}}-$ трехмерный оператор

Лапласа, $U_{j}(j=1,2)$ - потенциалы, описывающие взаимодействие волн на границе раздела сред:

$U_{1}=\left(\alpha_{1} \psi_{1}+\beta \psi_{2}\right) \delta(z), U_{2}(x)=\left(\alpha_{2} \psi_{2}+\beta \psi_{1}\right) \delta(z),(3)$

$\alpha_{j}(j=1,2), \beta$ - параметры, определяющие характер взаимодействия рассматриваемых волн с границей раздела сред и между собой. Форма таких потенциалов неоднократно использовалась при форму- лировке моделей для описания особенностей взаимодействия линейных волн в условиях двухканального рассеяния [9].

Поскольку нас будут интересовать воны, распространяющиеся вдоль границы раздела сред, то волновые функции представимы в виде: $\psi_{j}(x, y, z)=\psi_{j}(z) \exp \left(i k_{x} x+i k_{y} y\right), j=1,2$. Тогда решение уравнений (1) и (2) с потенциалами (3) сводится к решению стационарных НУШ:

$$
\begin{gathered}
\frac{1}{2 m_{j}} \psi_{j}^{\prime \prime}(z)+\varepsilon_{j} \psi_{j}(z)+\gamma_{j}(z)\left|\psi_{j}(z)\right|^{2} \psi_{j}(z)=0, \\
j=1,2,
\end{gathered}
$$

с граничными условиями:

$$
\begin{gathered}
\psi_{j}(+0)=\psi_{j}(-0)=\psi_{j}(0) ; j=1,2, \\
\left\{\begin{array}{c}
\left.\psi_{1}^{\prime}(+0)-\psi_{1}^{\prime}(-0)\right\}=2 m_{1}\left\{\alpha_{1} \psi_{1}(0)+\beta \psi_{2}(0)\right\} \\
\left.\psi_{2}^{\prime}(+0)-\psi_{2}^{\prime}(-0)\right\}=2 m_{2}\left\{\alpha_{2} \psi_{2}(0)+\beta \psi_{1}(0)\right\} .
\end{array}\right.
\end{gathered}
$$

В (4) введены обозначения:

$$
\varepsilon_{1}=E-\frac{k_{x}^{2}+k_{y}^{2}}{2 m_{1}}, \varepsilon_{2}=E-\Omega-\frac{k_{y}^{2}}{2 m_{2}}-\frac{\left(k_{x}-k_{0}\right)^{2}}{2 m_{3}} .
$$

В рассматриваемом случае решения уравнений (4) при положительных параметрах нелинейности, удовлетворяющие граничным условиям (5) и (6), можно искать в виде:

$$
\psi_{j}(z)=\left\{\begin{array}{l}
\frac{A_{j}^{(+)}}{\operatorname{ch} q_{j}\left(z-z_{1}\right)}, z>0, \\
\frac{A_{j}^{(-)}}{\operatorname{ch} q_{j}\left(z-z_{2}\right)}, z<0,
\end{array}\right.
$$

где параметры $z_{i}(i=1,2)$ определяют положения центров возбуждений (двух связанных солитонов) по разные стороны от границы раздела сред.

Подстановка (7) в уравнения (4) позволяет получить амплитуды локализации:

$$
A_{j}^{( \pm)}=q_{j}\left(m_{j} \gamma_{j}^{( \pm)}\right)^{-1 / 2} j=1,2,
$$

а также выражения:

$$
\begin{gathered}
q_{1}^{2}=k_{x}^{2}+k_{y}^{2}-2 m_{1} E, \\
q_{2}^{2}=k_{y}^{2}+\frac{m_{2}}{m_{3}}\left(k_{x}-k_{0}\right)^{2}+2 m_{2}(\Omega-E) .
\end{gathered}
$$

Если принять $k_{x}=k \cos \varphi, k_{x}=k \sin \varphi$, то (9) и (10) перейдут в:

$$
q_{1}^{2}=k^{2}-2 m_{1} E,
$$

$$
q_{2}^{2}=k^{2} \sin ^{2} \varphi+\frac{m_{2}}{m_{3}}\left(k \cos \varphi-k_{0}\right)^{2}+2 m_{2}(\Omega-E) .
$$

Отсюда следует связь волновых чисел:

$$
a k^{2}+2 b k+c=0,
$$




$$
\begin{gathered}
a=1-\frac{m_{1}}{m_{2}}+m_{1}\left(\frac{1}{m_{2}}-\frac{1}{m_{3}}\right) \cos ^{2} \varphi, \\
b=\frac{m_{1}}{m_{3}} k_{0} \cos \varphi, c=\frac{m_{1}}{m_{2}}\left(q_{2}^{2}-k_{0}^{2}\right)-q_{1}^{2}-2 m_{1} \Omega .
\end{gathered}
$$

В частности, (13) упрощается в предельном случае, когда $k_{0}=0$, откуда выражается волновое число: $k^{2}=-c / a$. Данные зависимости определяют значения энергии при фиксированном направлении волнового вектора, то есть при фиксированном угле $\varphi$. Иначе, данные соотношения можно интерпретировать с другой стороны, при фиксированной энергии они позволяют определять направления волнового вектора волны, локализованной вблизи границы раздела сред.

Подстановка (7) в граничные условия (5) и (6) приводит к соотношениям, связывающим параметры среды, дефекта и возбуждения:

$$
\begin{gathered}
\gamma_{j}^{(+)} \operatorname{ch}^{2} q_{j} z_{2}=\gamma_{j}^{(-)} \operatorname{ch}^{2} q_{j} z_{1}, j=1,2, \\
4 m_{1} m_{2} \beta^{2}=\Delta_{1} \Delta_{2}, \\
\Delta_{j}=2 m_{j} \alpha_{j}-q_{j}\left(\text { th } q_{j} z_{1}-\operatorname{th} q_{j} z_{2}\right), j=1,2 .
\end{gathered}
$$

Воспользовавшись соотношениями (14), величинам (16) можно придать вид, исключающий $z_{2}$ :

$$
\begin{gathered}
\Delta_{j}=2 m_{j} \alpha_{j}- \\
-q_{j}\left\{\text { th } q_{j} z_{1}-\left[1-\left(1-t^{2} q_{j} z_{1}\right) \gamma_{j}^{(+)} / \gamma_{j}^{(-)}\right]^{1 / 2}\right\}, \\
j=1,2 .
\end{gathered}
$$

Если рассматривать только локализованные состояния, когда положения центров возбуждений расположены симметрично относительно дефекта, то есть когда $z_{2}=-z_{1}=-z_{0}$, и одинаковый положительный ангармонизм по обе стороны от дефекта $\left(\gamma_{j}^{(+)}=\gamma_{j}^{(-)}\right)$, то соотношение (14) выполняется автоматически, а из (15) получится выражение:

$$
m_{1} m_{2} \beta^{2}=\left(m_{1} \alpha_{1}-q_{1} \text { th } q_{1} z_{0}\right)\left(m_{2} \alpha_{2}-q_{2} \text { th } q_{2} z_{0}\right) \text {. (18 }
$$

Из (18) при отсутствии взаимодействия ветвей закона дисперсии волны на границе раздела сред, когда $\beta=0$, следует, что они становятся независимыми, причем: $m_{j} \alpha_{j}=q_{j}$ th $q_{j} z_{0}, j=1,2$. Такие выражения совпадают с точностью до обозначений с соотношением между параметрами, полученными в [10]. Следовательно, учет взаимодействия ветвей закона дисперсии на границе раздела приводит к видоизменению области существования нелинейных локализованных волн.

В случае малой энергий локализации волн, когда $q z_{0}<<1$, из (18) можно получить положение центра:

$$
z_{0}=\frac{m_{1} m_{2}\left(\alpha_{1} \alpha_{2}-\beta^{2}\right)}{m_{2} \alpha_{2} q_{1}^{2}+m_{1} \alpha_{1} q_{2}^{2}} .
$$

Наибольший интерес представляет определение возможности существования несимметрично локализованного вблизи раздела сред состояния. Исследуем возможность реализации такой ситуации, предположив выполнения условия $z_{2}=0$. Тогда из (14) получается выражение th $q_{j} z_{1}=\eta_{j}$, где обозначено $\eta_{j}=\left(1-\gamma_{j}^{(-)} / \gamma_{j}^{(+)}\right)^{1 / 2}, j=1,2$, откуда следует, что

$$
q_{2}=\xi q_{1},
$$

где $\xi=\operatorname{Arch}\left(\gamma_{2}^{(+)} / \gamma_{2}^{(-)}\right)^{1 / 2} / \operatorname{Arch}\left(\gamma_{1}^{(+)} / \gamma_{1}^{(-)}\right)^{1 / 2}$.

Это означает, что различие амплитуд локализации характеризуется отношением параметров нелинейностей сред. В результате из (15) получаются выражения для волнового числа $q_{1( \pm)}=\mu \pm\left(\mu^{2}-v\right)^{1 / 2}$, где $\mu=\frac{\eta_{1} m_{2} \alpha_{2}+\xi \eta_{2} m_{1} \alpha_{1}}{\xi \eta_{1} \eta_{2}}$, $v=4 m_{1} m_{2}\left(\alpha_{1} \alpha_{2}-\beta^{2}\right) / \xi \eta_{1} \eta_{2}$. Отсюда следует, что если выполняется требование $\beta_{c}<\beta<\left(\alpha_{1} \alpha_{2}\right)^{1 / 2}$, где $\beta_{c}^{2}=\alpha_{1} \alpha_{2}-\frac{\left(\eta_{1} m_{2} \alpha_{2}+\xi \eta_{2} m_{1} \alpha_{1}\right)^{2}}{4 \xi \eta_{1} \eta_{2} m_{1} m_{2}}$, то существует два положительных решения.

Если $\beta>\left(\alpha_{1} \alpha_{2}\right)^{1 / 2}$, то существует одно положительное решение $q_{1(+)}=\mu+\left(\mu^{2}+|v|\right)^{1 / 2}>0$ и одно отрицательное $q_{1(-)}=\mu-\left(\mu^{2}+|v|\right)^{1 / 2}<0$. При $\beta<\beta_{c}$ корни $q_{1 \pm}$ становятся комплексными.

Таким образом, если выбрать управляющим параметром величину интенсивности энергии взаимодействия на границе раздела сред $\beta$, то при его изменении в пространстве параметров системы можно получать различные типы локализованных состояний несимметричного вида. При фиксированных значениях параметров следует выбрать нужный корень для определения волнового числа и энергии локализации, который будет далее обозначаться $\kappa=q_{1}$.

В случае слабого различия нелинейности сред, когда значение $\xi$ близко к единице, получается:

$$
\kappa=\frac{2 m_{1} m_{2}\left(\alpha_{1} \alpha_{2}-\beta^{2}\right)}{\eta_{1} m_{2} \alpha_{2}+\xi \eta_{2} m_{1} \alpha_{1}} .
$$

В предельных случаях выражения для волнового числа $k$ принимают простой вид. В частности, при $k_{0}=0$ из (13) для несимметричного состояния с учетом (19) получается:

$$
k^{2}=\frac{2 m_{2} \Omega+\left(1-\xi^{2}\right) \kappa^{2} m_{2} / m_{1}}{m_{2} / m_{1}-1+\left(1-m_{2} / m_{3}\right) \cos ^{2} \varphi} .
$$

В другом предельном случае, когда $k_{0} \neq 0$ и $m_{1}=m_{2}=m_{3}=m$ из (13) получается: 


\title{
C. Е. САВОТЧЕНКО
}

$$
k=\frac{2 m \Omega+k_{0}^{2}+\left(1-\xi^{2}\right) \kappa^{2}}{2 k_{0} \cos \varphi} .
$$

Также представляет интерес двумерный случай, когда $\varphi=0$ при $k_{0} \neq 0$ и $m_{1}=m_{3} \neq m_{2}$, что соответствует волне с $k_{y}=0$. Из (13) тогда получается:

$$
k=\frac{m_{1}\left(2 m_{2} \Omega+k_{0}^{2}\right)+\kappa^{2}\left(m_{2}-m_{1} \xi^{2}\right)}{2 k_{0} m_{2}} .
$$

В другом предельном случае, также соответствующем двумерной системе, когда $\varphi=\pi / 2$ при $k_{0} \neq 0$ и $m_{1} \neq m_{2}$, и определяющим волну с $k_{x}=0$, из (13) возможно выразить:

$$
k^{2}=\frac{m_{1}\left(2 m_{2} \Omega+k_{0}^{2}\right)+\kappa^{2}\left(m_{2}-m_{1} \xi^{2}\right)}{m_{2}-m_{1}} .
$$

Ясно, что при определенных соотношениях между параметрами можно добиться того, что выражения (20) и (23) дают вещественные значения волнового числа $k$. Данный анализ провести несложно и он аналогичен проделанному в работе [9].

Таким образом, в отличие от несвязных локализованных солитонных состоянии, описанных в [10], полученные в данной работе результаты показывают, что в зависимости от ориентации волнового вектора волны по отношению к выделенным направлениям изоэнергетической поверхности могут возникать локализованные состояния как симметричные относительно границы раздела нелинейных сред, так и несимметричные, энергии которых лежат в широком диапазоне.

\section{СПИСОК ЛИТЕРАТУРЫ}

1. Ахмедиев Н. Н., Корнеев В. И., Кузьменко Ю. В. // ЖЭТФ, 1985, т. 88, № 1, с. 107-115.

2. Горшков К. А., Островский Л. А., Папко В. В. // ЖЭТФ, 1976, т. 71, № 2(8), с. 585-593.

3. Кившарь Ю. С., Косевич А. М., Чубыкало О. А. // ЖЭТФ, 1987, т. 93, № 3(9), с. 968-977.

4. Kivshar Yu. S., Kosevich A. M., Chubykalo O. A. // Phys. Rev. A. 1990, vol. 41, №. 3, pp. 1677-1688.

5. Савотченко С. Е. // Известия высших учебных заведений. Физика, 2004, т. 47, № 5, с. 79-84.

6. Савотченко С. Е. // Вестник Воронежского государственного университета. Серия: Физика. Математика, 2016, № 4, с. 51-59.

7. Герасимчук И. В., Ковалев А. С. // Физика низких температур, 2000, т. 26, № 8, с. 799-809.

8. Abdullaev F. Kh., Baizakov B. B., Umarov B. A. // Optics Communications, 1998, vol. 156, pp. 341-346.

9. Косевич А. М., Мацокин Д. В. // Физика низких температур, 2000, т. 26, № 6, с. 615-619.

10. Богдан М. М., Герасимчук И. В., Ковалев А. С. // Физика низких температур, 1997, т. 23, № 2, c. $197-207$.

\section{INTERACTION OF THE LOCALIZED STATES NEAR NONLINEAR MEDIA BORDER}

\section{(C) 2017 S. E. Savotchenko}

\author{
Belgorod State Technological University named after V. G. Shukhov, 46 Kostyukova str., \\ 308012 Belgorod, Russia \\ e-mail:savotchenkose@mail.ru
}

Received 24.01.2017

\begin{abstract}
In this paper the coupled states that are localized near the nonlinear boundary media and propagating along it are considered. The boundary of nonlinear media with different parameters of anharmonicity of interatomic interaction creates a disturbance of medium characteristics, which is focused at distances much smaller than the width of the localization of the propagating waves. It is expected that the particle has a complex linear law of dispersion with several branches different parameters in a model proposed in this paper. The problem is reduced to the solution of nonlinear Schrцdinger equations with boundary conditions of a special kind. Explicit solutions of nonlinear Schrцdinger equations with positive nonlinear parameters satisfying the boundary conditions were found.

The relations which at a fixed energy allow determining the directions of the wave vector of the wave localized near the boundary were derived

It is shown that taking into account the interaction of the branches of the dispersion law at the boundary leads to the modification of the domain of existence of nonlinear localized waves. The possible existence of nonlinear localized states which are asymmetric in relation to the media boundary was
\end{abstract}


founded. These states are described by wave functions dampening moving to a distance from the boundary. They have a soliton-like profile in the direction perpendicular to the boundary. The structure and shape of such states are determined by the sign of interaction anharmonism in the medium and by the intensity of the excitation interaction and other characteristics of the medium. The equations determining the energy of the wave localized along the media boundary for a fixed direction of its wave vector are derived. Dependences of the wave numbers from the parameters of the system for localized states in various private cases are explicitly expressed. A two-dimensional case determined by a fixed value of the wave vector direction along the boundary of nonlinear medium is also considered. It is shown that if to select a control parameter the value of the interaction energy intensity at the boundary then at its changing in the system of parameters one can get different types of localized states of an asymmetric type.

Keywords: Nonlinear Schrцdinger equation, plane defect, soliton, localized states, nonlinear waves.

\section{REFERENCES}

1. Ahmediev N. N., Korneev V. I., Kuzmenko U. V. Journal of Experimental and Theoretical Physics, 1985, vol. 88, no. 1, pp. 107-115. (in Russia)

2. Gorshkov K. A., Ostrovskij L. A., Papko V. V. Journal of Experimental and Theoretical Physics, 1976, vol. 71, no. 2(8), pp. 585-593. (in Russia)

3. Kivshar U. S., Kosevich A. M., Chubykalo O. A. Journal of Experimental and Theoretical Physics, 1987, vol. 93, no. 3(9), pp. 968-977. (in Russia)

4. Kivshar U. S., Kosevich A. M., Chubykalo O. A. Phys. Rev. A., 1990, vol. 41, no. 3, pp. 1677-1688. DOI: https://doi.org/10.1103/PhysRevA.41.1677

5. Savotchenko S. E. Russian Physics Journal, 2004, vol. 47, no. 5, pp. 79-84. (in Russia)
6. Savotchenko S. E. Bulletin of Voronezh State University. Series: Physics. Mathematics, 2016, no. 4, pp. 51-59. Available at: http://www.vestnik.vsu.ru/pdf/ physmath/2016/04/2016-04-05.pdf

7. Gerasimchuk I. V., Kovalev A. S. Low Temperature Physics, 2000, vol. 26, no. 8, pp. 81-83.

8. Abdullaev F. Kh., Baizakov B. B., Umarov B. A. Optics Communications, 1998. vol. 156, pp. 341-346. https://doi.org/10.1016/S0030-4018(98)00451-9

9. Kosevich A. M. Low Temperature Physics, 2000, vol. 23, no. 6, pp. 615-619.

10. Bogdan M. M., Gerasimchuk I. V., Kovalev A. S. Low Temperature Physics, 1997, vol. 23, no. 2, pp. 197207.
Савотченко Сергей Евгеньевич - д. ф. - м. н, профессор, Белгородский государственный технологический университет имени В. Г. Шухова; тел.: +7(920) 5610446, e-mail: savotchenkose@mail.ru
Savotchenko Sergey E. - Dr. Sci. (Phys.-Math.), Professor, Belgorod State Technological University named after V. G. Shukhov; ph.: +7(920)5610446, e-mail: savotchenkose@mail.ru 